\title{
Metrological Confirmation of Measurement Equipment
}

\author{
Ling REN, Qiaoli LV, Rui ZHANG, \\ Xiaorong LEI, Yunpeng REN, Ying ZHANG
}

Metrology Department, Shaanxi Applied Physics-Chemistry Institute, Xi'an, 710061, China

Key words: Measurement Equipment; Measurement Process Control; Metrological Confirmation

\begin{abstract}
The metrological confirmation of measurement equipment was discussed in this paper. The relationship between the accuracy of measurement equipment and the data issued was analyzed. The important significance for the metrological confirmation of measurement equipment in the measurement and control system was clarified.
\end{abstract}

\section{Introduction}

In order to ensure measurement quality, measurement standards and measurement instrument or equipment should be controlled, in addition measurement process should also be controlled. A complete set of measurement and control system should be established for measurement process control. Measuring and control system was consisted of two aspects: metrological confirmation of measurement equipment and measurement process control. The so-called measurement process was a set of resources, activities and influence quantities, which were interrelated and related with implementation of measurement. Resources included surveyor, measurement equipment, measurement procedure, measurement method, etc. Influence quantities were various factors caused by environmental conditions, which affected the measurement results. The input of measurement process was devices under test, measuring equipment, surveyor, etc. The output of the measurement process was the calibration certificate or report. Measurement process control was the measure to be considered as a process, which was monitoring and analysis of data obtained by the measurement process and taking corrective action. So that the inaccuracy of the measurement process was within the technical requirements specified. By measurement process control, each element of the measurement process can be controlled, such as standard, equipment, environment, personnel, methods and so on, in order to prevent errors. Accurate measuring device or not was related to the accuracy of the data which was issued. That directly affected the quality of products. Therefore, metrological confirmation of measurement equipment had an important significance in the measurement and control system.

\section{The Metrological Confirmation of Measurement Equipment}

The so-called metrological confirmation was a set of operations, which ensure the measuring device meet the expected requirements. Firstly, the confirming work was to determine the metrological characteristics of measurement equipment. That included measurement parameters, measuring range, uncertainty of measurement or the maximum permissible error. Some measurement equipment included resolution, stability, etc. There were many methods to determine the metrological characteristics of measurement equipment, such as periodic verification (calibration), statistical analysis and so on. Secondly, we must confirm whether measurement equipment met the requirements for intended use. If it met its specifications but not the requirements for product testing, the measurement equipment could not be confirmed using. For some measurement equipment, such as the common power supply, an oscilloscope and so on, which was only used for functional check not for issue data, was performed a functional check without periodic testing or calibration. And the metrological confirmation interval could be extended. The premise was that measurement equipment could meet the requirements of product testing. In other words, the measurement equipment would not affect the quality of the product. Metrological 
confirmation generally included calibration and verification, the necessary adjustments or repairs and subsequent recalibration, the results compared with the metrological requirements intended use of the equipment, and sealing and labeling required (state flag). When measurement equipment had been proven suitable for the intended use and documented, metrological confirmation was only completed. The expected applications included measurement range, resolution, maximum permissible errors, etc. Measurement requirements, which were not specified in product requirements, were usually different from product requirements. The concept of metrological confirmation was a series of activities such as calibration, adjustment, repair, verification, seals and other labels for measurement equipment, also including verification, contrast, etc. The purpose of the activity was to ensure that measurement equipment met needs in the state. All measurement equipment could not always kept within a certain error, which in use would be shifted over time. In order to maintain its original accuracy after a certain time, measurement equipment must be carried out a series of activities such as calibration, commissioning or repair, recalibration to seal or mark, etc. Through these activities, the measurement equipment met the accuracy requirements of the intended use for a long period of time. As could be seen from the definition, metrological confirmation was the activities carried out to meet the requirements of the measurement equipment.

\section{The Method of Measurement Confirmation}

(1) Clearly defined responsibilities and authorities of personnel for device management and measurement assurance.

(2) Figured out testing requirements of measurement equipment for the product, which was conducted technical review.

(3) Established traceable records of management, maintenance, use and metrological confirmation procedures for measurement equipment.

(4) Established measurement equipment ledger, which usually include equipment type, serial number, technical specifications, conclusions, etc.

(5) Draw up calibration specifications for measurement equipment (If applicable).

(6) Formulated metrological confirmation mark, and color-coded it in which state usually.

(7) Under specified conditions measurement equipment met its technical indicators. And the impact of influence quantities should be carried out to assess.

(8) External measurement equipment must be subjected to metrological confirmation before putting into use.

\section{Metrological Confirmation Intervals}

Considerations: specifications of measurement equipment, use conditions of measurement equipment, correction value of influence quantity, records of use and maintenance for measurement equipment, testing requirements of product, trend f measurement equipment (usually obtained from the calibration data). If there was something wrong with measurement equipment, risk analysis was necessary. Metrological confirmation interval could be changed, which was adjusted according to the product testing requirements and the use of measurement equipment.

When product testing requirements increased or technical specifications of measurement equipment decreased(such as stability), metrological confirmation interval should be shortened. Conversely when product testing requirements decreased or technical specifications of measurement equipment was very stable, metrological confirmation interval could be extended.

\section{The Adjustment Method of Metrological Confirmation Interval}

(1) Automatic adjustment or staged adjustment. When metrological confirmation was in accordance with an established interval, measurement equipment was within the allowable error limit. And then metrological confirmation interval could be extended. If measurement equipment was without the allowable error limit, metrological confirmation interval should be shortened. Due 
to unpredictable circumstances, before metrology did metrological confirmation, equipment could not be used out of the predetermined metrological confirmation interval.

(2) Control chart. Calibration results of measurement equipment at a calibration point were sequentially drawn on the control chart. Metrological confirmation interval could be adjusted in accordance with fitting calculated based on those points and its trends analyzed.

(3) Countdown. The measurement equipment was connected to a timer and countdown. Once the timer reaches a predetermined time, the measurement equipment should be calibrated and re-confirmed. This method was automatically extended metrological confirmation interval for infrequently used measurement equipment.

(4) Verification standard. Measurement equipment which was confirmed periodically was verified with a good stability verification standard. When abnormal data was found, measurement equipment should be recalibrated and re-confirmed immediately.

\section{Measurement Equipment Verification and Comparison within the Period of Validity}

When measurement equipment was being used, according to their importance and frequency of use, the metrology department could take surveillance measures such as during verification, comparison, etc. According to actual usage in the metrological confirmation cycle, ensured that the measurement equipment had been in use to meet the anticipated requirements of the controlled state. Some substandard measurement equipment was due to these causes such as: seal integrity compromised in use, logo breakage or loss, function suspicious behavior, overload or malfunction. Since its metrological confirmation was still within the validity period, measurement equipment could also be confirmed by Metrology department authorized qualified personnel with the verification standard, comparison, etc.

\section{References}

[1] Nation Technic Intendance Office, Metrology Management, Deng Zheng, Liang Chunyu, Beijing: China Metrology Press, 1997.

[2] Wang zhengke, Internet Technology, Beijing: Tsinghua University Press, 1998. 